\title{
Multivariate fMRI Approaches to Flexible Sensorimotor Maps in Parietal Cortex
}

\author{
๑Deborah A. Barany, ${ }^{1,2}{ }^{\circledR}$ Allison D. Shapiro, ${ }^{1}$ and Taraz G. Lee ${ }^{1}$ \\ ${ }^{1}$ Department of Psychological and Brain Sciences and ${ }^{2}$ Interdepartmental Program in Dynamical Neuroscience, University of California, Santa Barbara, \\ Santa Barbara, California 93106 \\ Review of Haar et al.
}

The posterior parietal cortex (PPC) is uniquely suited to coordinate goal-related action because of its rich connectivity with frontal motor regions and posterior sensory regions. Among other functions, PPC is essential for computing sensorimotor transformations that require perceptual representations to guide goal-related motor behavior. To achieve this, specialized areas within PPC are thought to integrate distributed multimodal sensory inputs into cohesive spatial maps of the workspace (Filimon, 2010). From these maps, PPC may encode multiple flexible representations of a movement plan to be further processed by frontal motor areas (Sabes, 2011).

Visuomotor adaptation paradigms can help decipher the role of PPC in sensorimotor transformations by experimentally manipulating the relationship between visual and movement space. In standard tasks, visual feedback is transiently distorted (e.g., rotated by a fixed angle) to induce novel mappings between motor plans and perceptual feedback. After a few misreaches, subjects reliably adapt to the distorted feedback to regain high accuracy. Once the distortion is removed, aftereffects on subsequent reaches suggest that subjects have indeed encoded a new or altered visuomotor map (Wolpert et al.,

Received June 16, 2015; revised July 22, 2015; accepted July 24, 2015. Correspondence should be addressed to Deborah A. Barany, Department of Psychological and Brain Sciences, University of California, Santa Barbara, Santa Barbara, CA 93106. E-mail: barany@dyns.ucsb.edu. DOI:10.1523/JNEUROSCI.2307-15.2015

Copyright $\odot 2015$ the authors $\quad 0270-6474 / 15 / 3511763-03 \$ 15.00 / 0$
2011). Human and nonhuman primate research has implicated PPC as a key player within a larger network of regions that facilitate this visuomotor adaptation. However, isolating the specific role of PPC activity is particularly challenging because activity may correspond to newly learned mappings or to multiple pre-existing sensorimotor mappings.

In a recent issue of The Journal of $\mathrm{Neu}$ roscience, Haar et al. (2015) addressed this question by examining whole-brain directional selectivity with multivariate pattern analysis (MVPA) of fMRI data collected while human subjects performed a visuomotor adaption task. In contrast to traditional univariate analyses that average activity across relatively large brain regions, MVPA offers a refined framework that can reveal information represented by fine patterns of spatial activation. This allows researchers to decode cognitive representations from brain activity and to dissociate between neural mappings that would otherwise show the same overall level of activation. The ubiquity of user-friendly software packages for MVPA has provided researchers with a new lens through which to examine their fMRI data (Haxby, 2012). Importantly, these analyses yield nuanced distinctions that are fundamentally different from standard statistical approaches based on the general linear model and thus warrant careful consideration in their interpretation.

Haar et al. (2015) implemented a slow event-related fMRI design in which par- ticipants made out-and-back hand movements to one of four visual targets. All movements were performed on a small MRI-compatible tablet that provided the participants online cursor feedback and recorded precise movement kinematics. The visual information varied across three sequential phases of trials. In the baseline phase, cursor feedback matched the actual movement direction. In the critical rotation phase, a $45^{\circ}$ counterclockwise rotation was introduced to separate the visual target and cursor feedback from the movement. A final "washout" phase removed the rotation so that the visual feedback again matched the actual movement.

Using complementary region-of-interest and searchlight analyses, Haar et al. (2015) tested whether movement direction could be decoded from single-trial multivoxel response patterns. For each condition, they trained a classifier to predict direction on all but one of the trials and then used it to predict the direction of the omitted trial. Overall classification accuracy was calculated as the percentage of correctly decoded directions over all trials. In all conditions, the authors found significant above-chance classification of movement direction in early visual cortex (Vis), dorsal premotor cortex (PMd), supplementary motor area (SMA), primary motor cortex (M1), anterior cerebellum $(\mathrm{aCB})$, and both the superior parieto-occipital cortex (SPOC) and medial intraparietal sulcus (mIPS) within PPC. 
Although these results identify regions whose activity shows direction specificity during visuomotor tasks, they do not reveal what aspects of the task drive this specificity. Accurate within-condition decoding could have resulted from a classifier using any combination of visual (target location/ online cursor feedback), motor (movement vector/goal location), or proprioceptive (arm position) task features. More stringent generalization (cross-classification) tests that probe whether local activity patterns elicited by one context generalize to other contexts can help distinguish which task features steer classification. This approach has been particularly fruitful in discovering the underlying components of movement from fMRI response patterns (Eisenberg et al., 2011; Ogawa and Inui, 2012; Barany et al., 2014; Krasovsky et al., 2014). The authors' generalization approach consisted of training a classifier to dissociate movement direction in baseline trials and using that classifier to predict direction on the rotated trials in two independent generalization tests. The classifier was "correct" if it predicted (1) the rotated visual direction or (2) the actual movement direction. They found generalization in Vis and SPOC to the rotated visual direction and generalization in PMd, SMA, $\mathrm{M} 1$, and $\mathrm{aCB}$ to the movement direction. These results align closely with the proposed functional roles for these regions in visually guided movement (Andersen and Cui, 2009; Gallivan and Culham, 2015). mIPS was the only region to exhibit accurate within-condition classification without also generalizing from the baseline to rotated trials.

Since the task used by Haar et al. (2015) involved four possible targets, the nature of the misclassifications could potentially reveal important characteristics about neural coding within a region, as any "incorrect" predictions might be systematically biased. For example, on each rotated trial, the classifier might frequently predict a target that is $45^{\circ}$ from the visual direction but is in line with $\left(0^{\circ}\right.$ from $)$ the movement direction. Plotting the classifier's predictions as a function of distance from the visual or motor directions yielded tuning curve-like histograms [Haar et al. (2015), their Fig. 6B]. Assessing the peak of these distributions allowed the authors to infer whether the classifier was relying more on visual or motor information. In Vis and SPOC, the classifier most frequently predicted the visual direction, whereas for PMd, SMA, M1, and $\mathrm{aCB}$, the classifier most frequently predicted the motor direction. Consistent with the generalization analysis, mIPS did not show significant tuning, although the two most frequently predicted directions were the rotated visual and the actual motor directions.

Haar et al. (2015) suggested that the lack of generalization in mIPS (a null result) provides evidence that mIPS stores new visuomotor mappings as a result of adaptation. Although this interpretation is in line with proposed contributions of PPC to visuomotor adaptation, there are equally plausible alternative explanations to consider given the limitations of MVPA in FMRI and the known functional organization of PPC. MVPA often cannot accurately decode intermixed neural representations within one region and, likewise, within a particular voxel (Gallivan and Culham, 2015). Thus, MVPA would not be able to detect or distinguish between two pre-existing mappings that encode conflicting information during the rotation phase. Suppose there are two separate neural populations in mIPS: one coding the visual target (or online feedback) and one coding the movement direction to the target (or proprioceptive estimates). A classifier trained on baseline trials in which the directions for these representations are congruent could make use of any weighted combination of the neural populations to accurately decode the direction of baseline or washout trials. On rotated trials, the neural populations represent two different directions ( $45^{\circ}$ apart), but the classifier still operates under the assumption that they are identical. Each generalization prediction may therefore be arbitrarily driven toward either the visual or movement direction based on which of the two neural populations contributed slightly more to the classifier's model for that particular trial. This could lead to the tuning curve results seen in mIPS, where both the rotated visual and actual movement directions exhibited abovechance, but not significant, tuning.

Null classification in mIPS might also be related to the feedback control mechanisms and contextual modulation of sensory feedback used to improve motor performance (Diedrichsen et al., 2005). In this case, the feedback control necessary to drive movements in the rotation condition would be different than in the baseline condition, leading to chance generalization.

A third potential source for the observed null classification result in mIPS on rotated trials is that the relative neural response patterns for visual and motor features vary across the time course of a single trial (Shen and Alexander, 1997). Given the temporal resolution of the BOLD signal, this would be difficult to detect without explicitly separat- ing each phase of a trial in the experimental design. Using a similar visuomotor rotation paradigm, Ogawa and Inui (2012) separated the target, delay, and execution stages. In this work, mIPS was found to encode movement features, but only before the execution stage. Area V6A (i.e., SPOC) encoded visual features during target presentation, as in the study by Haar et al. (2015), but switched to encoding the movement features during the delay period.

It is important to note that none of these alternative hypotheses necessitate a fundamental change in how neural populations respond during visuomotor adaptation relative to baseline. Rather, null classification may be simply attributable to the classifier's failure to detect invariant features between the baseline and rotated conditions.

Haar et al. (2015) have convincingly shown that mIPS, in addition to multiple brain regions along the motor hierarchy, can dissociate between reaches to different directions and that coding in MIPS is not specific to only the visual or motor components of the task. A key avenue for future research is to resolve the extent to which PPC encodes new visuomotor mappings relative to pre-existing maps after adaptation to altered feedback. Other multivariate approaches, such as representational similarity analysis and forward encoding models, can detect mixed representations within regions and may be useful to assess specific models of underlying neural representations within PPC (Eisenberg et al., 2011; Serences and Saproo, 2012). Future work using these techniques will help us gain a deeper understanding of how the human motor system adapts to an ever-changing environment.

\section{References}

Andersen RA, Cui H (2009) Intention, action planning, and decision making in parietalfrontal circuits. Neuron 63:568-583. CrossRef Medline

Barany DA, Della-Maggiore V, Viswanathan S, Cieslak M, Grafton ST (2014) Feature interactions enable decoding of sensorimotor transformations for goal-directed movement. J Neurosci 34:6860-6873. CrossRef Medline

Diedrichsen J, Hashambhoy Y, Rane T, Shadmehr R (2005) Neural correlates of reach errors. J Neurosci 25:9919-9931. CrossRef Medline

Eisenberg M, Shmuelof L, Vaadia E, Zohary E (2011) The representation of visual and motor aspects of reaching movements in the human motor cortex. J Neurosci 31:1237712384. CrossRef Medline

Filimon F (2010) Human cortical control of hand movements: parietofrontal networks for reaching, grasping, and pointing. Neuroscientist 16:388-407. CrossRef Medline 
Gallivan JP, Culham JC (2015) Neural coding within human brain areas involved in actions. Curr Opin Neurobiol 33:141-149. CrossRef Medline

Haar S, Donchin O, Dinstein I (2015) Dissociating visual and motor directional selectivity using visuomotor adaptation. J Neurosci 35: 6813-6821. CrossRef Medline

Haxby JV (2012) Multivariate pattern analysis of fMRI: the early beginnings. Neuroimage 62 : 852-855. CrossRef Medline

Krasovsky A, Gilron R, Yeshurun Y, Mukamel R
(2014) Differentiating intended sensory outcome from underlying motor actions in the human brain. J Neurosci 34:15446-15454. CrossRef Medline

Ogawa K, Inui T (2012) Reference frame of human medial intraparietal cortex in visually guided movements. J Cogn Neurosci 24:171182. CrossRef Medline

Sabes PN (2011) Sensory integration for reaching: models of optimality in the context of behavior and the underlying neural circuits. Prog Brain Res 191:195-209. CrossRef Medline
Serences JT, Saproo S (2012) Computational advances towards linking BOLD and behavior. Neuropsychologia 50:435-446. CrossRef Medline

Shen L, Alexander GE (1997) Neural correlates of a spatial sensory-to-motor transformation in primary motor cortex. J Neurophysiol 77: 1171-1194. Medline

Wolpert DM, Diedrichsen J, Flanagan JR (2011) Principles of sensorimotor learning. Nat Rev Neurosci 12:739-751. CrossRef Medline 